\title{
Responsabilité personnelle et neurosciences
}

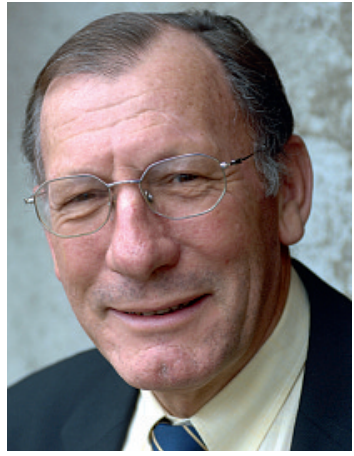

Jean Martin
Le libre arbitre est une des grandes questions philosophiques, depuis John Locke parmi d'autres. Les connaissances nouvelles des neurosciences amènent derechef à se demander dans quelle mesure nous agissons de manière vraiment autonome ou sommes déterminés par des données d'origine externe ou interne - sur lesquelles nous n'avons pas barre. Doctrine et pratique judiciaires pourraient être bouleversées si la notion de responsabilité pénale du sujet agissant librement était mise en cause. Un ouvrage issu d'une réunion de juristes et spécialistes des neurosciences patronnée par l'Association américaine pour l'avancement de la science (AAAS) est une référence utile [1]. Ci-dessous quelques extraits (ma traduction) et commentaires.

D'abord, une remarque de Greely sur la question de savoir ce qu'est, fondamentalement, je/moi: «Il se peut que je sois mon esprit, que je sois mon cerveau bien plus que je ne suis mes gènes. Mes gènes ne sont pas moi. Mais mon esprit, mon cerveau, c'est peut-être moi. Cela étant, si beaucoup pensent que leur esprit est l'essence de qui ils sont, il pourrait y avoir moins de résistance à évaluer les gens à l'aide de données neuroscientifiques qu'il n'y en a à les évaluer par des données génétiques.» Question liée à la problématique classique nature-culture (naturenurture). A noter aussi l'éventualité, selon Ramachandran, «que notre esprit conscient ne soit pas complètement libre de dire Je veux $>$ mais le soit de dire 〈Je ne veux pas»». Le libre arbitre pourrait ainsi être un pouvoir de veto ...?

Sur la signification des données récentes des neurosciences (Gazzaniga et Steven): «A notre avis, les neurosciences ne peuvent offrir que fort peu à la compréhension de la responsabilité, aucun pixel sur un scan du cerveau ne pourra jamais démontrer la culpabilité ou la non-culpabilité [...]. L'idée de responsabilité est une construction sociale et existe dans les règles de la société, elle n'est pas dans la structure neuronale du cerveau.» Il ne faut pas à leur avis s'attendre à un affaiblissement de la notion de responsabilité pénale: «Il importe de réaliser que, finalement, même si la causalité/origine d'un acte (criminel ou autre) peut s'expliquer en termes de fonctionnement du cerveau, cela ne signifie pas que la personne devrait être innocentée. L'évidence scientifique suggère que notre cerveau dirige nos actions mais pas notre personnalité. Alors que le cerveau peut être vu comme un instrument plus ou moins automatique, comme une montre, en tant que personnes nous sommes habituellement libres de choisir notre propre destinée» (à ce sujet, voir aussi [2, 3]).

Une autre idée est que le cadre sociétal sera en mesure de tenir compte des connaissances nouvelles sans abandonner ses principes ni perdre sa pertinence (qu'il se montrera résilient, en quelque sorte): «Les développements des neurosciences pourraient bien avoir un impact substantiel sur la manière dont la loi voit les gens et leurs comportements, mais le système légal devrait être capable d'assimiler et d'utiliser une science, même révolutionnaire, sans bouleverser sa structure fondamentale» (Garland).

Commentaire sur une évolution qui jouera un rôle dans les prétoires: «Des institutions sociales majeures, depuis l'école et les tribunaux jusqu'aux lieux de travail et au système de santé, bénéficieront grandement de tests dérivés de la recherche en neurosciences. Les tests sont devenus un way of life dans la culture américaine contemporaine». Toutefois, en rapport avec ces tests, «il y a une tendance forte à réduire beaucoup de problèmes à des critères soi-disant solides et objectifs. Ce type de réductionnisme a deux avantages. D'abord les données sont plus concrètes; ensuite des tests biologiques insatisfaisants suggèrent une suite facile, à savoir renvoyer ceux qui en sont affligés au système de santé» (Tancredi). Il importe à l'évidence d'être attentif à ne pas abuser d'une telle objectivisation et médicalisation des problèmes sociaux ou de situations pénales.

Verrons-nous un flou croissant, voire une confusion, quant aux appréciations que la société - telle que nous la connaissons - doit faire des actions de ses membres et à la responsabilité personnelle y relative à la faveur de données des neurosciences? Comme on le voit à propos de génétique: la presse, scientifique comme grandpublic, fait état souvent déjà de traits particuliers, de conduites hors norme, qui pourraient être liés au bagage héréditaire? Je ne serais pas surpris que ces interrogations se multiplient.

Jean Martin, membre de la rédaction et membre de la Commission nationale d'éthique 